8

\title{
Determination of Herbicides in Human Urine by Liquid Chromatography-Mass Spectrometry With Electrospray Ionization
}

\author{
Isabel C. S. F. Jardim, Joseane M. Pozzebon, and Sonia C. N. Queiroz
}

\begin{abstract}
Summary
A method for the determination of triazines (simazine, atrazine) and their metabolite 2-chloro-4,6-diamino-1,3,5-triazine by liquid chromatography-mass spectrometry with electrospray ionization (LC-MS/ESI) in human urine is described. The method outlines the sample preparation, which involves protein precipitation with acetonitrile and solidphase extraction using C18 cartridges, and the qualitative and quantitative chromatographic analyses. The method may be used to assess occupational exposure to triazine herbicides following the urinary excretion of low levels of both the parent compounds and their metabolites.
\end{abstract}

Key Words: Atrazine; 2-chloro-4,6-diamino-1,3,5-triazine; human urine; liquid chromatography-mass spectrometry with electrospray ionization; simazine; solid-phase extraction; triazines.

\section{Introduction}

Occupational exposure to triazine herbicides results in urinary excretion of low levels of both the parent compounds and their metabolites (1-6). Quantitative and qualitative analyses require highly sensitive and specific purification and determination procedures.

The increasing use of liquid chromatographic (LC) methods for pesticide analysis is because of their applicability in determining thermally labile compounds and polar compounds, which require derivatization before gas chromatographic (GC) analysis (7). The good compatibility of aqueous samples with reversed-phase liquid chromatography contributes to the fact that LC is frequently applied for analyses of environmental and biological fluid samples. Although ultraviolet (UV) detectors are the most common choice for LC, on-line liquid chromatography with mass spectrometry (LCMS) is an interesting approach because conventional LC detectors can cause false- 


\begin{tabular}{lll} 
& \\
Herbicide & $\begin{array}{c}\text { Substituents in positions } 4 \text { and } 6 \\
\mathrm{R}_{1}\end{array}$ \\
\hline $\begin{array}{l}\text { 2-chloro-4,6-diamino-1,3,5-triazine } \\
\text { Simetabolite) }\end{array}$ & $-\mathrm{H}_{2}$ \\
Atrazine & $-\mathrm{C}_{2} \mathrm{H}_{5}$ & $-\mathrm{H}_{2}$ \\
\hline
\end{tabular}

Fig. 1. Chemical structures of the herbicides studied.

positive results. This technique combines the advantages of coupling the separation power of LC to the unequivocal identification potential of MS for the determination of pesticides in biological samples $(8,9)$.

This work describes a method for the determination by liquid chromatographymass spectrometry with electrospray ionization (LC-MS/ESI) of the triazines simazine and atrazine and their metabolite 2-chloro-4,6-diamino-1,3,5-triazine (Fig. 1), present in human urine.

\section{Materials}

1. Standard grade simazine ( $>98 \%)$, atrazine $(>97 \%)$, and the metabolite 2-chloro-4,6diamino-1,3,5-triazine $(>96 \%)$.

2. Methanol, pesticide grade.

3. Acetonitrile, HPLC grade.

4. Chloroform, pesticide grade.

5. Water purified by a Milli-Q Plus System (Millipore, Bedford, MA).

6. Envi C18 Supelclean 3-mL extraction tubes (e.g., Supelco, Bellefonte, PA).

7. Urine samples. These must be kept in the freezer at $-20^{\circ} \mathrm{C}$ until use. The samples are stable for $15 \mathrm{~d}$ (see Note 1).

8. LC-MS system equipped with an injector with a $10-\mu \mathrm{L}$ loop and a UV detector (e.g., Waters, Milford, MA).

9. Chromatographic column $(150 \times 3.9 \mathrm{~mm}$ id $)$ and guard column $(20 \times 3.9 \mathrm{~mm}$ id $) \mathrm{C} 18$ (e.g., Waters Nova-Pak C18, $4 \mu \mathrm{m}$ ).

10. Vacuum extraction system (manifold) (e.g., Supelco).

\section{Methods}

The steps described below outline (1) sample preparation and (2) chromatographic analysis.

\subsection{Sample Preparation}

Urine samples are frozen at $-20^{\circ} \mathrm{C}$ until use. After the urine samples are thawed, they must be shaken for homogenization. The required volume must be sampled as quickly as possible to avoid sedimentation of solids. 


\subsubsection{Removal of Proteins}

1. Take a $2-\mathrm{mL}$ volume of urine.

2. Basify the sample by addition of $200 \mu \mathrm{L}$ of $\mathrm{NH}_{4} \mathrm{OH}(0.01 \%)(\mathrm{pH} \sim 9.0)$ and add $4 \mathrm{~mL}$ of acetonitrile at room temperature.

3. Centrifuge the mixture ( $5 \mathrm{~min}, 3000 \mathrm{~g}$ ).

4. Take a $3-\mathrm{mL}$ aliquot of the supernatant containing urine-acetonitrile $(1: 2 \mathrm{v} / \mathrm{v})$ and dilute with $20 \mathrm{~mL}$ Milli-Q water (see Note 2).

\subsubsection{Cleanup Using Solid-Phase Extraction}

The cleanup is made by solid-phase extraction using Envi C18 Supelclean extraction tubes $(3 \mathrm{~mL})$ and a vacuum extraction system (manifold). The steps that follow are involved.

\subsubsection{Sorbent Conditioning}

Rinse the cartridge with $10 \mathrm{~mL}$ methanol and then with $5 \mathrm{~mL}$ Milli-Q water for equilibration. Avoid allowing the sorbent to dry; otherwise, the recovery will be decreased.

\subsubsection{Sample Application}

Apply all the diluted sample (Subheading 3.1.1.) to the cartridge, under vacuum, at a flow rate of $3 \mathrm{~mL} / \mathrm{min}$.

\subsubsection{WASHING/Removal OF INTERFERENCES}

Remove undesired matrix components by passing $5 \mathrm{~mL}$ Milli-Q water through the cartridge. This eluate is discarded, and the sorbent bed is then dried under vacuum for $1 \mathrm{~min}$.

\subsubsection{Elution and Concentration of the Sample}

Wipe the delivery needles of the manifold and place labeled collection tubes under the cartridges. With the vacuum off, add the elution solvent, $3 \mathrm{~mL}$ chloroform, to each cartridge (see Note 3). Turn on vacuum and carefully open the tap of the manifold to initiate elution of the analyte. The elution must be carried out slowly to obtain suitable recovery of the analyte.

The organic layer is evaporated to dryness under a stream of nitrogen, and the residue is dissolved in $200 \mu \mathrm{L}$ of acetonitrile. The preconcentration factor is fivefold because the volume applied to the cartridge corresponds to $1 \mathrm{~mL}$ of initial urine sample, and the final volume is $200 \mu \mathrm{L}$.

\subsection{Chromatographic Analysis}

\subsubsection{Standard Solution Preparation}

Stock solutions of simazine, atrazine, and the metabolite 2-chloro-4,6-diamino1,3,5-triazine are prepared in methanol at concentrations of $100 \mu \mathrm{g} / \mathrm{mL}$. Intermediate concentrations are prepared in mobile phase at concentrations of $1000 \mu \mathrm{g} / \mathrm{L}$ of each herbicide (see Note 4). These solutions are stored in the refrigerator at $4^{\circ} \mathrm{C}$, at which they are stable for at least $60 \mathrm{~d}$. Further solutions at five different concentrations (5, $30,60,100$, and $200 \mu \mathrm{g} / \mathrm{L}$ ) of each analyte are prepared daily for construction of the analytical curve. 


\subsubsection{Spiked Samples}

Because certified reference material is not available and there are insufficient samples for intercomparison assays, method accuracy is determined using spiked samples. Three different concentrations $(40,60$, and $80 \mu \mathrm{g} / \mathrm{mL})$ of each pesticide are spiked into known volumes of pesticide-free urine (blank) to calculate recovery. After spiking the samples, carry out procedures as in Subheadings 3.1.1. and 3.2.1.

\subsubsection{Chromatographic Conditions}

The mobile phase is acetonitrile: $\mathrm{H}_{2} \mathrm{O}(40: 60 \mathrm{v} / \mathrm{v})$ with the $\mathrm{pH}$ of the mobile phase adjusted to 3.0 with $0.1 \% \mathrm{CH}_{3} \mathrm{COOH}$ (see Notes 5 and $\mathbf{6}$ ). The mobile phase flow rate is set at $0.3 \mathrm{~mL} / \mathrm{min}$. The column is directly coupled to the inlet of a chromatograph with a quadrupole MS system operated using the ESI source. Measurements are carried out using the positive ESI mode. All measurements are carried out at room temperature (see Note 7).

For optimization of the MS parameters, each compound is dissolved in $\mathrm{pH} 3.0$ mobile phase and injected separately. A source temperature of $150^{\circ} \mathrm{C}$ is used. Nitrogen is used as both nebulizer and drying gas at flow rates of 30 and $300 \mathrm{~L} / \mathrm{h}$, respectively.

The capillary voltage is $25 \mathrm{~V}$ for the determination of these herbicides and their metabolite. For identification, the instrument is operated in the total ion mode; for quantification, acquisition is in the selected ion monitoring (SIM) mode. The selected ions for quantification are 145, 202, and 216 for 2-chloro-4,6-diamino-1,3,5-triazine, simazine, and atrazine, respectively (see Note 8).

\subsubsection{Qualitative and Quantitative Analyses (see Note 9)}

1. Inject the following sequence: solvent (mobile phase), matrix blank (urine without pesticide), standards, solvent, spiked samples (urine with spiked pesticides), and samples. Matrix blank, spiked samples, and samples are injected after processing according to the steps in Subheadings 3.1.1. and 3.1.2. The standards, spiked samples, and samples are all injected in triplicate.

2. Compare the chromatograms. No peak should be detected at the retention times of the pesticides in the chromatograms of solvent and matrix blank.

3. Determine the limit of detection (LOD) and the limit of quantification (LOQ).

4. Construct the analytical curve with the areas obtained from the chromatograms vs concentration. Linear analytical curves $\left(r^{2} \geq 0.99\right)$ must be obtained in the range from LOQ to $200 \mu \mathrm{g} / \mathrm{L}$.

5. Calculate the recoveries by comparing the replicate responses of extracted spiked samples with those of standards that represent $100 \%$ recovery. Recovery is calculated using the following equation:

Recovery $(\%)=($ Mass of analyte after extraction $\times 100) /$ Mass of analyte added

6. Calculate the precision in terms of repeatability using the results obtained from the recovery samples.

7. If peaks are detected in the chromatograms of the samples, confirm the presence of the herbicide by comparing both retention time and the mass spectra using full-scan mode.

8. Quantify the corresponding amount of the herbicide by the external standard method using the linear equation obtained from the analytical curve. 


\section{Notes}

1. The stabilities of the herbicides in urine with storage time are evaluated by monitoring aliquots of urine having two different concentrations of simazine and atrazine (80, 150 $\mu \mathrm{g} / \mathrm{L})$ and submitting them to replicate freeze/thaw cycles (10). The concentrations of the herbicides are determined after the initial thawing (zero time) and after thawing at successive prefixed times (once each week). Each concentration is compared with the mean of the zero time (initial concentration). Degradation values of $<10 \%$ are obtained over a period of $15 \mathrm{~d}$.

2. The sample is diluted to decrease solution strength before the extraction procedure.

3. The C18 Supelclean extraction tubes are eluted with methanol and compared with those eluted with chloroform. Recovery is generally good for both solvent systems, but backgrounds are cleaner using chloroform. The evaporation time for chloroform eluate is also shorter than that for methanol. Therefore, the use of chloroform as an elution solvent is recommended for the triazines under study.

4. An analytical curve prepared using mobile phase as solvent is preferable for dissolving the components of the sample, which is a prerequisite for using LC.

5. The $\mathrm{pH}$ of the mobile phase is adjusted using a calibrated $\mathrm{pH}$ meter with glass and thermal compensation electrodes.

6. A prerequisite in LC-MS is that the analyte must be ionizable in solution, so the mobile phase often contains a small amount of a volatile acid or base. If such additives impair the chromatographic separation, they can be added after the separation, before the eluent enters the ESI interface. The separation of the triazine herbicides and their metabolite is tested at various compositions of the eluent and at different $\mathrm{pH}$ values. Because of their polar character, the triazines do not interact strongly with the $\mathrm{C} 18$ reversed phase, the most utilized stationary phase in LC. Thus, it is necessary to add acetic acid to the mobile phase to increase the molecular ionization of the compounds, which also improves the detection in analysis by MS.

7. Quantification can also be performed by LC with UV detection. The UV detector is set at $220 \mathrm{~nm}$ as a compromise between the maximum absorbance of the analytes and the reduced background of the eluents at this wavelength (11). Acetonitrile is chosen as an organic modifier in the mobile phase owing to its low absorbance background in the UV region.

8. After defining the conditions for SIM of the triazines, the product ions are recorded with a single quadrupole set at a fixed $m / z$ value representing $[\mathrm{M}+\mathrm{H}]^{+}$. A urine sample is a complex matrix consisting of various components; therefore, it is essential to use an extraction procedure to remove these interferents. However, using SIM, no problem is encountered in the quantification of the analytes because only the ion corresponding to each compound is selectively monitored.

9. The method must be validated using the instrumentation with which the analyses will be made. According to our results for validation of this method (12), analytical curves are obtained using standard solutions of the triazines, and they showed good linearity in the range from LOQ to $800 \mu \mathrm{g} / \mathrm{L}$ with correlation coefficients $>0.999$. The following values are easily obtained for the validation parameters: average recoveries ranging from 82 to $114 \%$; of $0.4 \mu \mathrm{g} / \mathrm{L} \mathrm{LOD} ; 1.3 \mu \mathrm{g} / \mathrm{L}$ LOQ; and precision values of $0.5 \%$ (repeatability) and $2.4 \%$ (intermediate precision). These values are considered acceptable for biological samples (11). 


\section{Acknowledgments}

We acknowledge FAPESP for financial support and a fellowship (to J. M. P.). We also thank C. H. Collins and W. Vilegas for helpful discussions and suggestions.

\section{References}

1. Mendas, G., Drevenkar, V., and Zupancic-Kralj, L. (2001) Solid-phase extraction with styrene-divinylbenzene sorbent for high-performance liquid or gas chromatographic determination of urinary chloro- and methylthiotriazines. J. Chromatogr. A 918, 351-359.

2. Catenacci, G., Barbieri, F., Bersani, M., Fereoli, A., Cottica, D., and Maroni, M. (1993) Biological monitoring of human exposure to atrazine. Toxicol. Lett. 69, 217-222.

3. Mendas, G., Tkalcevic, B., and Drevenkar, V. (2000) Determination of chloro- and methylthiotriazine compounds in human urine: extraction for gas chromatographic analysis with nitrogen-selective and electron capture detection. Anal. Chim. Acta 424, 7-18.

4. Perry, M. J., Christiani, D. C., Mathew, J., Degenhart, D., Tortorelli, J., Strauss, J., and Sonzogni, W. C. (2000) Urinalysis of atrazine exposure in farm pesticide applicators. Toxicol. Ind. Health 16, 285-290.

5. Catenacci, G., Maroni, M., Cottica, D., and Pozzoli, L. Assessment of human exposure to atrazine through the determination of free atrazine in urine. Bull. Environ. Contam. Toxicol. 44, 1-7.

6. Barr, D. A. and Needham, L. (2002) Analytical methods for biological monitoring of exposure to pesticides: a review. J. Chromatogr. B 778, 5-29.

7. Hogendoorn, E. and van Zoonen, P. (2000) Recent and future developments of liquid chromatography in pesticide trace analysis. J. Chromatogr. A 892, 435-453.

8. Careri, M., Mangia, A., and Musci, M. (1996) Applications of liquid chromatographymass spectrometry interfacing systems in food analysis: pesticide, drug and toxic substance residues. J. Chromatogr. A 727, 153-184.

9. Henion, J., Brewer, E., and Rule, G. (1998) Sample preparation for LC/MS/MS: analyzing biological and environmental samples. Anal. Chem. 70, 650A-656A.

10. Causon, R. (1997) Validation of chromatographic methods in biomedical analysis. Viewpoint and discussion. J. Chromatogr. B 689, 175-180.

11. Sacchero, G., Apone, S., Sarzanini, C., and Mentasti, E. (1994) Chromatographic behaviour of triazine compounds. J. Chromatogr. A 668, 365-370.

12. Pozzebon, J. M., Vilegas, W., and Jardim, I. C. S. F. (2003) Determination of herbicides and a metabolite in human urine by liquid chromatography-electrospray ionization mass spectrometry. J. Chromatogr. A 987, 375-380. 